\title{
Tác động của các yếu tố marketing tại điểm bán đến hành vi mua hàng tùy hứng thực phẩm đóng gói tại các điểm bán lẻ ở Thành phố Hồ Chí Minh
}

\section{The impact of point-of-sale marketing on impulse buying behaviour of packaged foods at retailers in Ho Chi Minh City}

\author{
Dương Hữu Hạnh ${ }^{1 *}$, Kiều Anh Tài ${ }^{1}$ \\ ${ }^{1}$ Trường Đại học Mở Thành phố Hồ Chí Minh, Việt Nam \\ *Tác giả liên hệ, Email: duonghuuhanh@gmail.com
}

THÔNG TIN

DOI:10.46223/HCMCOUJS. econ.vi.16.3.983.2021

Ngày nhận: 21/09/2020

Ngày nhận lại: 15/10/2020

Duyệt đăng: 04/12/2020

\section{Tù khóa:}

marketing tại điểm bán; mua hàng tùy hứng; nhận biết thương hiệu; trưng bày sản phẩm; vật phẩm quảng cáo tại điểm bán

\section{TÓM TÁT}

Trong điều kiện cạnh tranh ngày càng gay gắt, các điểm bán lẻ không chỉ đơn thuần là một nơi phân phối, mua bán hàng hóa mà còn là nơi mà cả nhà bán lẻ và nhà sản xuất tiến hành các hoạt động marketing để gây ảnh hưởng lên quyết định của người mua hàng. Nghiên cứu này xem xét tác động của marketing tại điểm bán lên hành vi mua hàng tùy hứng. Nghiên cứu đã tiến hành khảo sát, sử dụng với mẫu thuận tiện, với khách hàng đã mua một món hàng thực phẩm đóng gói cụ thể tại một cửa hàng bán lẻ ở Thành phố Hồ Chí Minh để dùng ngay tại chổ hay mang dùng trên đường đi. Dữ liệu thu thập được sau khi kiểm tra lọc thì có 302 mẫu được sử dụng để đánh giá thang đo và kiểm định mô hình bằng phân tích nhân tố khám phá và phân tích cấu trúc tuyến tính theo phương pháp bình phương tối thiểu từng phần. Kết quả nghiên cứu cho thấy các yếu tố trưng bày vật phẩm quảng cáo tại điểm bán, nhận biết thương hiệu, trưng bày sản phẩm có ảnh hưởng đến hành vi mua hàng tùy hứng thông qua lần lượt là cảm giác hứng thú với món hàng và sự thôi thúc mua hàng tùy hứng. Các hàm ý quản trị đã được thảo luận trong bài báo dựa trên kết quả nghiên cứu.

\section{ABSTRACT}

Nowadays, as competition becomes more and more intensified, a retail outlet is not just a venue for distribution or transaction but a promotional touchpoint for retailers and manufacturers vying to attract shoppers' attention and influence their impulsive behaviour. This study examines the impact of point-of-sale marketing on consumers' impulse buying behaviour.

Keywords:

The primary study was conducted using the survey method with a

impulse buying behavior; pointof-sale marketing; brand awareness; point-of-sale promotional materials; product displays packaged food at a retailer in Ho Chi Minh City for on-premise or on-the-go consumption. After screening, usable data, including 302 responses, was analyzed using EFA and PLS-SEM methods to assess scales and test hypotheses. The results showed that three 
point-of-sale marketing factors display point-of-sale materials; brand awareness and product display significantly influence impulse buying behaviour through sequentially the feeling of pleasure and the urge to buy impulsively. The managerial implications are also discussed.

\section{Giới thiệu}

Trong bối cảnh kinh doanh cạnh tranh gay gắt như hiện nay, bên cạnh các hình thức xúc tiến như giảm giá, khuyến mãi, nhà sản xuất và người bán lẻ còn phải chú ý các yếu tố marketing tại điểm bán lẻ như trưng bày bắt mắt ... để thu hút sự chú ý và tác động đến hành vi của khách hàng. Muốn vậy, người bán lẻ và người làm marketing cần thấu hiểu các hành vi mua hàng của người tiêu dùng và các tác nhân ảnh hưởng đến hành vi mua hàng tại điểm bán (Prashar, Parsad, Tata, \& Sahay, 2015). Việc xây dựng và hoàn thiện các hoạt động xúc tiến phù hợp, cập nhật thông tin cho khách hàng đúng thời điểm sẽ giúp các doanh nghiệp tăng hiệu quả marketing (Nguyen, 2014). Ngày nay, khái niệm marketing tại điểm bán hay marketing với người mua không còn xa lạ với các doanh nghiệp và nhà bán lẻ.

Trong lý thuyết liên quan đến hành vi người tiêu dùng từ lâu có một mảng nghiên cứu về hành vi mua hàng tùy hứng. Beatty và Ferrell (1998) đã định nghĩa hành vi mua hàng tùy hứng là hành động mua hàng đột nhiên và tức thì mà không có những ý định từ trước để mua sản phẩm cụ thể hoặc hoàn thành nhiệm vụ mua sắm. Với thực tiễn ngành bán lẻ khắp nơi không ngừng biến đổi thì loại hành vi khách hàng này không chỉ là mối quan tâm của người làm marketing mà của các nhà nghiên cứu; cụ thể như Amos, Holmes, và Keneson (2014), Santini, Ladeira, Vieira, Araujo, và Sampaio (2019), Kim, Lee, và Jung (2020), và Iyer, Blut, Xiao, và Grewal (2020). Hành vi mua hàng tùy hứng là một hiện tượng phức tạp, và thường có mối liên hệ đến những sự nhạy cảm cảm xúc (Rook, 1987). Tuy nhiên, các nghiên cứu trước đây về hành vi mua hàng tùy hứng chủ yếu thực hiện trong lĩnh vực bán lẻ thời trang (Park, Kim, \& Forney, 2006), nội thất, bán lẻ trong siêu thị (Zhou \& Wong, 2004), mỹ phẩm (P. T. Wu \& Lee, 2016) và gần đây là mua hàng tùy hứng qua mạng (L. Wu, Chen, \& Chiu, 2016). Nếu so với các mặt hàng như mỹ phẩm hay thời trang thì với những mặt hàng tiêu dùng nhanh thì khách hàng sẽ bị ảnh hưởng mạnh mẽ hơn bởi những yếu tố ngoại biên hơn là sự suy tính cẩn thận (Rossiter, Percy, \& Donovan, 1991). Nghiên cứu này nhận thấy có rất ít nghiên cứu công bố xem xét hành vi mua tùy hứng mà khách hàng ít bận tâm nhất như là mua một món hàng thực phẩm đóng gói để sử dụng tại chỗ hay mang dùng trên đường đi có thể bị ảnh hưởng nhiều bởi yếu tố hoàn cảnh.

Ngoài ra, các nghiên cứu trước đây phần lớn xem xét ảnh hưởng của bầu không khí tại điểm bán, các tín hiệu giác quan, các tác nhân bên trong của khách hàng, nhưng ít xem xét cụ thể các yếu tố marketing do người làm marketing và nhà bán lẻ triển khai tại điểm bán. Các nghiên cứu trước đây đã chỉ ra rằng có một số yếu tố marketing đóng vai trò kích thích các hành vi mua hàng một cách tùy hứng, không có dự định trước của người mua (Abratt \& Goodey, 1990; Mohan, Sivakumaran, \& Sharma, 2013). Các yếu tố đó liên quan đến điểm bán chẳng hạn như là vị trí trên kệ, giảm giá, dùng thử, trưng bày tại điểm bán. Chính vì vậy, nghiên cứu này nhằm xem xét tác động của các yếu tố marketing tại điểm bán, và quá trình tâm lý dẫn đến đến hành vi mua hàng tùy hứng thực phẩm đóng gói tại các điểm bán lẻ ở Thành phố Hồ Chí Minh (TPHCM), một trung tâm kinh tế thương mại của Việt Nam. Nghiên cứu này không chỉ đóng góp minh chứng giải quyết vấn đề hạn chế của lý thuyết về hành vi mua hàng, mà cũng đóng góp minh chứng từ một thị trường mới nổi là Việt Nam. Bối cảnh nghiên cứu là phù hợp với sự kêu gọi nghiên cứu cho mảng thị trường này để phát triển lý thuyết và đảm bảo 
ý nghĩa thực tiễn của lý luận (Sheth, 2011). Bên cạnh đó, thực tế công tác marketing của các doanh nghiệp ngành hàng tiêu dùng nhanh tại Việt Nam hiện nay vẫn chủ yếu tập trung vào các hoạt động truyền thông đại chúng và các hoạt động marketing tại điểm bán gần như chỉ được xem là cánh tay nối dài, thì nghiên cứu này có thể giúp các người làm marketing có thêm chứng cứ làm cơ sở đưa ra các giải pháp tăng cường các hoạt động marketing tại điểm bán để ảnh hưởng đến hành vi mua hàng tùy hứng của người mua, gia tăng cơ hội tiêu thụ sản phẩm, giúp tăng thêm hiệu quả marketing cho doanh nghiệp và hiệu quả bán hàng cho nhà bán lẻ.

\section{Cơ sở lý thuyết}

Nghiên cứu này được phát triển dựa trên nền tảng lý thuyết là mô hình Tác nhân kích thích (Stimuli) - Chủ thể (Organism) - Phản ứng (Respone) hay còn được gọi tắt là mô hình S-O$\mathrm{R}$ của Mehrabian và Russell (1974). Lý thuyết này cho rằng các tác nhân môi trường $(\mathrm{S})$ như màu sắc, nhiệt độ, cách bố trí sẽ tạo ra các phản ứng tâm lý $(\mathrm{O})$, từ đó các phản ứng tâm lý sẽ dẫn đến các hành vi phản ứng của con người $(R)$ với các tác nhân môi trường. Các nghiên cứu quốc tế trước đây đã lập luận cho sự phù hợp của lý thuyết này khi nghiên cứu hành vi mua hàng không có dự định trước. Cụ thể, Robert và John (1982) đã chỉ ra rằng, cảm giác hứng thú và cảm giác hưng phấn của người mua ngay tại điểm bán có tính quyết định với ý định mua hàng của khách hàng. Chang, Eckman, và Yan (2011) cũng dựa vào mô hình $\mathrm{S}-\mathrm{O}-\mathrm{R}$ để xem xét sự ảnh hưởng trực tiếp và gián tiếp của các nhân tố thuộc môi trường bán lẻ đến hành vi mua hàng tùy hứng. Tương tự, Chen và Lin (2018) xem xét trải nghiệm của các giác quan đến hành vi mua hàng tùy hứng. Dựa trên cơ sở lược khảo lý thuyết, nghiên cứu này sẽ vận dụng mô hình S-O-R để xem xét ảnh hưởng của các yếu tố marketing hoạt động tại điểm bán lẻ ( $\mathrm{S}$ ) đến phản ứng tâm lý $(\mathrm{O})$ và tiếp theo là phản ứng hành vi $(\mathrm{R})$. Nhiều nghiên cứu đã xem xét hành vi mua hàng tùy hứng chịu sự tác động của đặc điểm cá nhân của khách hàng (Weun, Jones, \& Beatty, 1998) hay loại sản phẩm (Inman, Winer, \& Ferraro, 2009; Jones, Reynolds, Weun, \& Beatty, 2003; Mishra, 2012). Nhưng đáng chú ý và có ý nghĩa thực tiễn hơn chính là sự xem xét ảnh hưởng của nhân tố tình huống (Beatty \& Ferrell, 1998) hay môi trường (Peck \& Childers, 2006) đến hành vi mua hàng tùy hứng, vì đây là những yếu tố mà người làm marketing có thể kiểm soát được.

\subsection{Các yếu tố marketing tại điểm bán}

Ngày nay, các nhà sản xuất không còn chỉ chú trọng các đòn bẩy marketing truyền thống (như là quảng cáo) để tạo ra nhận thức, thái độ mà còn tập trung sang đòn bẩy marketing để gây ảnh hưởng đến bước chốt quyết định mua hàng tại điểm bán; song song đó là những nhà bán lẻ đã tăng cường đầu tư vào hoạt động marketing tại điểm bán để kích thích các hành vi mua hàng không có kế hoạch (Bellini, Cardinali, \& Grandi, 2017). Một số nhà nghiên cứu cho rằng các yếu tố kích thích bên ngoài từ hoạt động marketing được xem là tác nhân chủ chốt của việc mua hàng tùy hứng (Iyer et al., 2020). Theo đó, những yếu tố marketing tại điểm bán lẻ đã được nghiên cứu trước đây và thực tiễn phổ biến trong các ngành hàng tiêu dùng nhanh phổ biến tại Việt Nam như trưng bày sản phẩm; trưng bày các vật phẩm quảng cáo, xúc tiến bán của nhà bán lẻ, sự nhận diện thương hiệu tại điểm bán sẽ được xét như là những tác nhân môi trường.

\subsection{Phản ứng tâm lý của chủ thể}

Theo lý thuyết S-O-R (Mehrabian \& Russell, 1974) các phản ứng tâm lý có thể là các cảm giác hứng thú (pleasure), hưng phấn (arousal), hay chế ngự (dominance). Trong đó, cảm giác hứng thú được xem xét xuyên suốt qua các bối cảnh nghiên cứu khác nhau từ siêu thị đến nhà hàng đến thức ăn đường phố đến dịch vụ (Bigné, Andreu, \& Gnoth, 2005; Choi, 2016; Ryu \& Jang, 2007). Cảm giác hứng thú được định nghĩa là những cảm giác như hứng khởi, thích thú, dễ chịu và kiêu hãnh (Bearden \& Netemeyer, 1999). Theo Coley và Burgess (2003), hành vi lướt 
qua khắp điểm bán có thể làm tăng trạng thái cảm xúc thì càng tăng khả năng người mua mua hàng tùy hứng hơn là dựa vào nhận thức hay suy tính.

Trưng bày sản phẩm là sự sắp xếp hàng hóa một cách hấp dẫn, ở những vị trí độc chiếm và dễ dàng tìm thấy sản phẩm thông qua hoạt động trưng bày (Parsad, Prashar, \& Tata, 2017). Các nghiên cứu cho việc trưng bày sản phẩm ở các khu vực có thể dễ dàng tiếp cận và nhìn thấy thúc đẩy khách hàng mua sắm tùy hứng thay vì mua theo kế hoạch đã định sẵn (Bandyopadhyay, 2016), giúp người mua hoàn thành nhanh chóng việc mua hàng của họ (Sorensen et al., 2017), có thể khiến người mua thấy dễ chịu hơn. Fiore, Yah, và Yoh (2000) cho rằng việc trưng bày sản phẩm có ảnh hưởng đến cảm giác hứng thú. Do đó, nghiên cứu đưa ra giả thuyết:

\section{H1: Trưng bày sản phẩm có tác động tích cực đến cảm giác hứng thú của khách hàng}

Các hoạt động xúc tiến của nhà bán lẻ bên trong cửa hàng đề cập đến các chương trình khuyến mãi như giảm giá, tặng kèm hàng và sự chủ động của nhân viên bán hàng tại điểm bán (Palazon \& Ballester, 2011). Bell, Corsten, và Knox (2011) tin rằng những người làm marketing có thể tạo ra hành động mua hàng không có kế hoạch bằng các lợi ích thêm dành cho người mua. Chang và cộng sự (2011) cho rằng nhân viên bán hàng biết hỗ trợ khách hàng bằng cách đặt câu hỏi cũng có thể gợi lên cảm xúc tích cực và từ đó kích thích mua sắm tùy hứng. Do đó, nghiên cứu đưa ra giả thuyết:

H2: Các hoạt động xúc tiến của điểm bán lẻ có tác động tích cực đến cảm giác hứng thú của khách hàng

Trưng bày vật phẩm quảng cáo tại điểm bán là trình bày những thứ chứa đựng các thông tin liên quan đến sản phẩm, thương hiệu và doanh nghệp trên quầy, kệ ... (Zhou \& Wong, 2004). Các vật phẩm quảng cáo tại điểm bán có hiệu quả kích thích hành vi mua hàng tùy hứng khi nó có thể thu hút khách hàng, gây được cảm giác thú vị và hiện đại với khách hàng (Zhou \& Wong, 2004). Poels và Dewitte (2008) cho rằng các quảng cáo in đặt tại điểm bán ảnh hưởng đến cảm giác hứng thú của khách hàng. Do đó, nghiên cứu đưa ra giả thuyết:

H3: Trung bày vật phẩm quảng cáo tại điểm bán có tác động tích cực đến cảm giác hứng thú của khách hàng

Nhận biết thương hiệu là sự nhận diện hay gợi nhớ thương hiệu của người tiêu dùng (Keller, Parameswaran, \& Jacob, 2011). Sự nhận biết thương hiệu sản phẩm hay có thể chỉ là một dấu hiệu như logo tại điểm bán cũng có thể giúp quyết định mua nhanh hơn, ít cân nhắc hơn, hay nói cách khác là hành vi mua hàng tùy hứng hơn. Aaker (1996) thì cho rằng sự nhận biết có tác động đến sự nhận thức và thái độ của khách hàng. Do đó, nghiên cứu đưa ra giả thuyết:

\section{H4: Nhận biết thương hiệu có tác động tích cực đến cảm giác hưng thú của khách hàng}

Nhiều nhà nghiên cứu lập luận rằng để hành vi mua hàng tùy hứng xảy ra thì phải tồn tại một trạng thái tâm lý nữa là sự thôi thúc mua một cách tùy hứng, một trạng thái mong muốn được trải nghiệm khi gặp một đối tượng trong môi trường; và trạng thái này rõ ràng diễn ra trước hành động tùy hứng thật sự mang tính tự phát và đột ngột (Beatty \& Ferrell, 1998; Rook \& Gardner, 1993). Leith và Baumeister (1996) đã phát hiện ra rằng cảm xúc sẽ thúc đẩy khách hàng tăng khả năng chấp nhận rủi ro. Theo Chang và cộng sự (2011), thôi thúc mua hàng tùy hứng là một loại của những khao khát không hợp lý và nó có thể được biểu hiện như là một ý định mua hàng tùy hứng. Do đó, nghiên cứu đưa ra giả thuyết:

\section{H5: Cảm giác hứng thú của khách hàng có tác động tích cực đến thôi thúc mua hàng} tùy hứng 


\subsection{Hành vi mua hàng tùy hứng}

Badgaiyan và Verma (2014) cho rằng thôi thúc mua hàng tùy hứng có tác động tích cực đến hành vi mua hàng tùy hứng. Thôi thúc mua hàng tùy hứng làm mất đi những sự lựa chọn thay thế, dẫn khách hàng đến hành vi mua hàng tùy hứng (Lee, 2008). Kacen và Lee (2002) cho rằng sự nhanh chóng quyết định mua hàng tùy hứng sẽ hạn chế những suy nghĩ hoặc cân nhắc kỹ càng giữa các lựa chọn. Do đó, nghiên cứu đưa ra giả thuyết:

H6: Thôi thúc mua hàng tùy hứng có tác động tích cực đến Hành vi mua hàng tùy hứng

\subsection{Mô hinh nghiên cứu}

Trên cơ sở lược khảo lý thuyết và các giả thuyết trên thì mô hình nghiên cứu được đề xuất như sau:

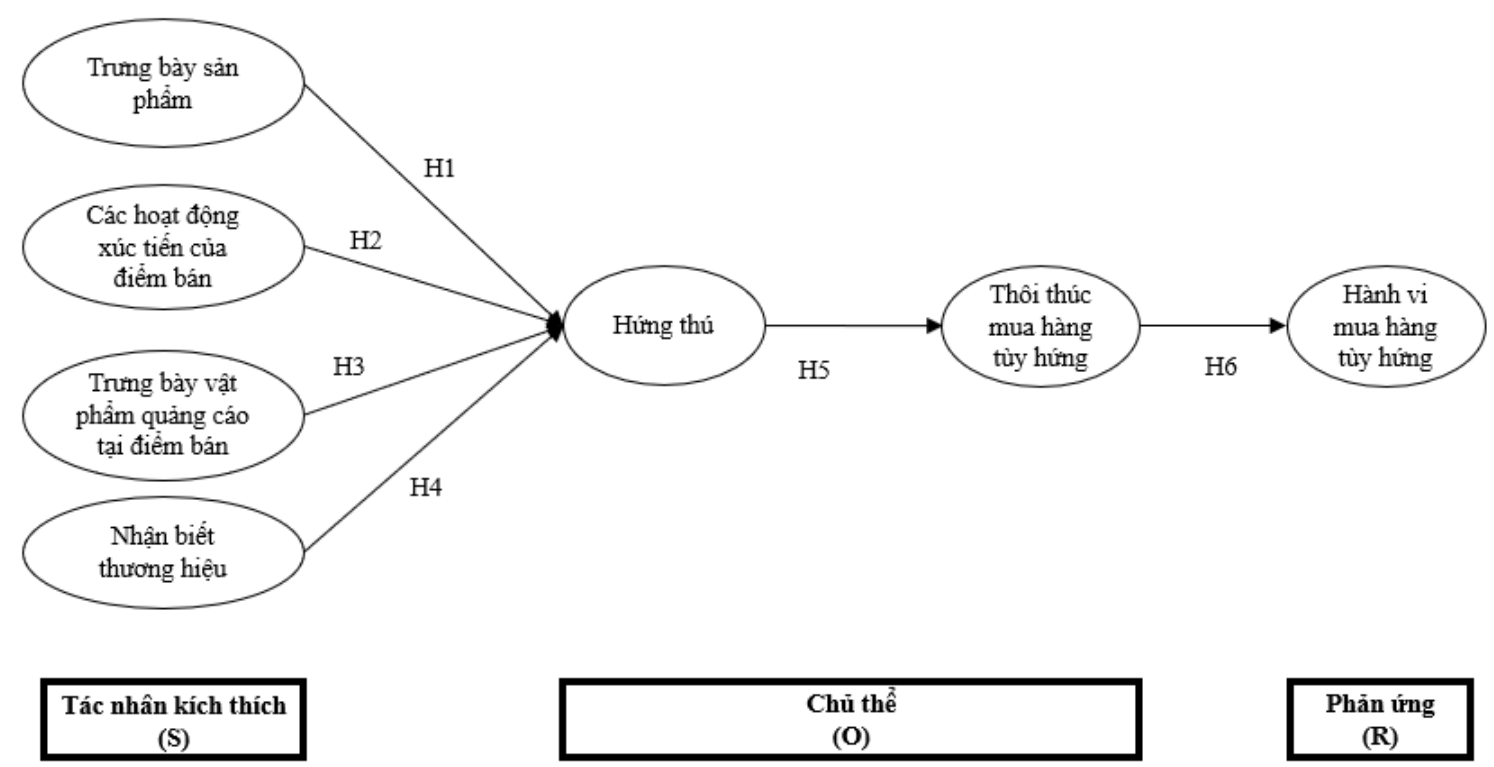

Hình 1. Mô hình nghiên cứu đề xuất

\section{Phương pháp nghiên cứu}

\subsection{Phwơng pháp nghiên cúu và thu thập dĩu liệu}

Phương pháp nghiên cứu chính là định lượng bằng hình thức khảo sát gửi bảng câu hỏi trực tiếp tới các khách hàng sau khi mua thực phẩm đóng gói tại các điểm bán lẻ như căn tin, cửa hàng tiện lợi ... trên địa bàn TPHCM sử dụng phương pháp lấy mẫu thuận tiện, và sử dụng bảng câu hỏi trực tuyến được xây dựng bằng công cụ Google Form gửi kèm email đến bạn bè, đồng nghiệp ... và để trên mạng xã hội. Theo Hair, Anderson, Babin, và Black (2010) thì số mẫu phải tối thiểu phải gấp năm lần số lượng biến quan sát thì kết quả nghiên cứu mới đảm bảo tính chính xác. Nghiên cứu được thực hiện với 25 biến quan sát, như vậy cần có tối thiểu 125 mẫu. Hair và cộng sự (2010) cũng gợi ý là mẫu từ 300 trở lên là khá lớn phù hợp để thực hiện nhiều loại phân tích cần thiết. Dữ liệu thu thập được sau khi làm sạch có 302 mẫu có thể sử dụng để phân tích.

\subsection{Bảng câu hỏi và thang đo}

Bảng câu hỏi bao gồm câu hỏi lọc, các thang đo và câu hỏi phân loại. Do các thang đo được sử dụng từ các nghiên cứu trước bằng tiếng Anh (Bảng 1) nên được dịch từ tiếng Anh sang tiếng Việt rồi dịch ngược lại, sau đó so sánh thang đo gốc và bản dịch ngược để kiểm tra tương đồng nội dung. Nội dung bảng câu hỏi được xây dựng sau khi thống nhất về nội dung của các thang đo, được khảo sát thử nghiệm để kiểm tra mức độ hiểu ý nghĩa câu chữ, hướng dẫn trước 
khi khảo sát đại trà. Đáp viên sẽ đánh giá mức độ với các nội dung của thang đo sử dụng thang Likert 5 bậc, với bậc 1 là hoàn toàn không đồng ý và bậc 5 là hoàn toàn đồng ý.

\section{Bảng 1}

Các thang đo sử dụng

\begin{tabular}{|l|l|}
\hline \multicolumn{1}{|c|}{ Khái niệm nghiên cứu } & \multicolumn{1}{c|}{ Nguồn } \\
\hline Trưng bày sản phẩm & Parsad và cộng sự (2017) \\
\hline Các hoạt động xúc tiến của điểm bán & Atulkar và Kesari (2018) \\
\hline Trưng bày vật phẩm quảng cáo tại điểm bán & Zhou và Wong (2004) \\
\hline Nhận biết thương hiệu & Aaker (1996) \\
\hline Cảm giác hứng thú & Choi (2016) \\
\hline Thôi thúc mua hàng tùy hứng & Beatty và Ferrell (1998) \\
\hline Hành vi mua hàng tùy hứng & Choi (2016) \\
\hline
\end{tabular}

Nguồn: Tác giả thực hiện (2020)

\subsection{Phân tích dữ liệu}

Với tập dữ liệu thu về, sau khi hoàn tất việc sàng lọc, kiểm tra, tổng hợp, mã hóa và làm sạch, sẽ tiến hành xử lý và phân tích số liệu bằng phần mềm SPSS 25 và Smart PLS 3.2.9. Nghiên cứu này tập trung vào đánh giá tác động chính của các yếu tố marketing và phân tích biến kết quả là hành vi mua hàng tùy hứng nên sử dụng Phương pháp bình phương tối thiểu từng phần áp dụng cho mô hình cấu trúc (PLS-SEM) là phù hợp (Nguyen \& Vu, 2020).

\section{Kết quả nghiên cứu}

\section{1. Đặc điểm mẫu nghiên cứu}

Bảng 2 thể hiện thông tin về mẫu nghiên cứu. Loại sản phẩm mà các đáp viên sử dụng nhiều hơn là nước giải khát với $72.2 \%$. Nữ chiếm $66.6 \%$ và theo độ tuổi thì nhóm từ 25 đến 34 tuổi chiếm tỷ lệ cao nhất là $46.4 \%$. Nhóm có thu nhập từ 05 đến dưới 10 triệu đồng chiếm tỷ lệ cao nhất với $36.8 \%$. Nhóm người có trình độ đại học chiếm $63.6 \%$, và có $64.2 \%$ đáp viên có tình trạng gia đình là độc thân.

\section{Bảng 2}

Thông tin về mẫu nghiên cứu

\begin{tabular}{|l|l|c|c|}
\hline \multicolumn{2}{|c|}{ Mẫu n $=\mathbf{3 0 2}$} & Tần số & \% \\
\hline \multirow{3}{*}{ Loại sản phẩm đã sử dụng } & Nước giải khát & 218 & 72.2 \\
\cline { 2 - 4 } & Thực phẩm đóng gói & 84 & 27.8 \\
\hline \multirow{3}{*}{ Giới tính } & Nam & 101 & 33.4 \\
\hline & Nũ̃ & 201 & 66.6 \\
\hline \multirow{3}{*}{ Tuổi } & Từ 18 đến 24 & 105 & 34.8 \\
\cline { 2 - 4 } & Từ 25 đến 34 & 140 & 46.4 \\
\cline { 2 - 4 } & Từ 35 đến 44 & 48 & 15.9 \\
\cline { 2 - 4 } & Từ 45 đến 54 & 9 & 3.0 \\
\hline
\end{tabular}




\begin{tabular}{|c|c|c|c|}
\hline \multicolumn{2}{|r|}{ Mẫu n = 302} & \multirow{2}{*}{$\begin{array}{c}\text { Tần số } \\
63\end{array}$} & \multirow{2}{*}{$\begin{array}{c}\mathbf{\%} \\
20.9 \\
\end{array}$} \\
\hline \multirow{5}{*}{ Thu nhập } & Dưới 05 Triệu VNĐ & & \\
\hline & Từ 05 Triệu đến dưới 10 Triệu VNĐ & 111 & 36.8 \\
\hline & Từ 10 Triệu đến dưới 20 Triệu VNĐ & 81 & 26.8 \\
\hline & Từ 20 Triệu đến dưới 30 Triệu VNĐ & 28 & 9.3 \\
\hline & Từ 30 Triệu VNĐ trở lên & 19 & 6.3 \\
\hline \multirow{4}{*}{ Học vấn } & Từ Phổ thông Trung học trở xuống & 7 & 2.3 \\
\hline & Cao đẳng, Trung cấp chuyên nghiệp & 21 & 7.0 \\
\hline & Đại học & 192 & 63.6 \\
\hline & Sau đại học & 82 & 27.2 \\
\hline \multirow{4}{*}{ Tình trạng hôn nhân } & Độc thân & 194 & 64.2 \\
\hline & Lập gia đình & 98 & 32.5 \\
\hline & Ly dị & 4 & 1.3 \\
\hline & Khác & 6 & 2.0 \\
\hline
\end{tabular}

Nguồn: Tác giả thực hiện (2020)

\section{2. Đánh giá thang đo bằng Cronbach's Alpha và EFA}

Các thang đo trong nghiên cứu này trước tiên được kiểm định Cronbach’s Alpha (Bảng 3) để loại các biến đo lường không phù hợp và đánh giá độ tin cậy của thang đo. Các biến có hệ số tương quan biến - tổng (Corrected Item - Total Correlation) nhỏ hơn 0.3 được xem xét loại bỏ. Kết quả kiểm định các thang đo đều có Cronbach's Alpha $>0.7$ và không có biến quan sát nào có hệ số tương quan biến tổng nhỏ hơn 0.3. Tuy nhiên, ở nhân tố Các hoạt động xúc tiến của điểm bán $(\mathrm{MA})$ có một biến thành phần là MA3 có hệ số tương quan biến tổng nhỏ nhất là 0.366 và hệ số Cronbach's Alpha nếu xóa biến sẽ là 0.823 . Với kết quả như trên, 25 biến quan sát tạm thời tiếp tục được đưa vào phân tích nhân tố khám phá (EFA) và sẽ xem xét biến quan sát MA3 ở các phân tích sau.

\section{Bảng 3}

Kết quả kiểm định Cronbach’s Alpha

\begin{tabular}{|l|c|c|c|}
\hline \multicolumn{1}{|c|}{ Thang đo } & $\begin{array}{c}\text { Số biến } \\
\text { quan sát }\end{array}$ & $\begin{array}{c}\text { Cronbach's } \\
\text { Alpha }\end{array}$ & $\begin{array}{c}\text { Hệ số tương quan biến } \\
\text { tổng nhỏ nhất }\end{array}$ \\
\hline Trưng bày sản phẩm (PD) & 3 & 0.720 & 0.463 \\
\hline $\begin{array}{l}\text { Các hoạt động xúc tiến của điểm } \\
\text { bán (MA) }\end{array}$ & 3 & 0.716 & 0.366 \\
\hline $\begin{array}{l}\text { Trưng bày vật phẩm quảng cáo tại } \\
\text { điểm bán (BV) }\end{array}$ & 4 & 0.874 & 0.686 \\
\hline Nhận biết thương hiệu (BA) & 5 & 0.845 & 0.600 \\
\hline Cảm giác Hứng thú (PL) & 4 & 0.815 & 0.511 \\
\hline Thôi thúc mua hàng tùy hứng (UI) & 3 & 0.840 & 0.675 \\
\hline Hành vi mua hàng tùy hứng (IB) & 3 & 0.838 & 0.616 \\
\hline
\end{tabular}

Nguồn: Kết quả phân tích bằng phần mềm SPSS 25 
Nghiên cứu tiếp tục thực hiện EFA cho các biến độc lập, các biến trung gian và biến kết quả, với phương pháp trích nhân tố là Principal Axis Factoring, phép xoay là Varimax và các biến quan sát có hệ số tải nhân tố nhỏ hơn 0.4 bị loại.

Kết quả EFA các biến độc lập cho thấy kiểm định Kaiser-Meyer-Olkin $(\mathrm{KMO})=0.845$ $(\geq 0.5)$ có ý nghĩa mẫu thích hợp để phân tích nhân tố và kiểm định Bartlett's với mức ý nghĩa thống kê (Sig. < 0.001$)$ có ý nghĩa các biến quan sát có mối tương quan với nhau trong tổng thể. Kết quả cho thấy, với Eigenevalue $>01$, trích được bốn nhân tố với tổng phương sai trích cho thấy giải thích được $57.514 \%$ sự biến thiên của dữ liệu. Kết quả EFA bốn nhân tố độc lập như Bảng 4. Các nhân tố bao gồm hầu hết các biến quan sát từ thang đo đã vận dụng, trừ một biến quan sát MA3 (Sự thân thiện của nhân viên) vốn là một biến quan sát của thang đo ban đầu Các hoạt động xúc tiến của điểm bán lại tải lên nhân tố Trưng bày vật phẩm quảng cáo và kết quả kiểm định Cronbach's Alpha ở trên cũng cho thấy nếu loại MA3 thì hệ số Cronbach's Alpha của biến Các hoạt động xúc tiến của điểm bán (MA) sẽ tăng và là 0.823 . Như vậy, sau khi thực hiện EFA, trên cơ sở xem xét giá trị nội dung và các định nghĩa khái niệm, thì nghiên cứu này loại biến quan sát MA3 ra khỏi thang đo của MA.

\section{Bảng 4}

Kết quả EFA từng nhân tố của các biến độc lập

\begin{tabular}{|c|c|c|c|c|c|}
\hline \multirow{2}{*}{\multicolumn{2}{|c|}{ Thành phần }} & \multicolumn{4}{|c|}{ Các nhân tố trích } \\
\hline & & \multirow{2}{*}{$\frac{1}{0.780}$} & \multirow[t]{2}{*}{2} & \multirow[t]{2}{*}{3} & \multirow[t]{2}{*}{4} \\
\hline \multirow{5}{*}{$\begin{array}{l}\text { Trưng bày vật phẩm quảng } \\
\text { cáo tại điểm bán }\end{array}$} & BV2 & & & & \\
\hline & BV3 & 0.774 & & & \\
\hline & BV1 & 0.744 & & & \\
\hline & BV4 & 0.726 & & & \\
\hline & MA3 & 0.540 & & & \\
\hline \multirow{5}{*}{ Nhận biết thương hiệu } & BA2 & & 0.775 & & \\
\hline & BA3 & & 0.764 & & \\
\hline & BA1 & & 0.730 & & \\
\hline & BA5 & & 0.671 & & \\
\hline & BA4 & & 0.645 & & \\
\hline \multirow{2}{*}{$\begin{array}{l}\text { Các hoạt động xúc tiến của } \\
\text { điểm bán }\end{array}$} & MA2 & & & 0.859 & \\
\hline & MA1 & & & 0.771 & \\
\hline \multirow{3}{*}{ Trưng bày sản phẩm } & PD2 & & & & 0.778 \\
\hline & PD1 & & & & 0.558 \\
\hline & PD3 & & & & 0.407 \\
\hline
\end{tabular}

Nguồn: Kết quả phân tích bằng phần mềm SPSS 25

EFA cho các biến trung gian cho thấy hệ số $\mathrm{KMO}=0.851(\geq 0.5)$ có ý nghĩa mẫu thích hợp để phân tích nhân tố và kiểm định Bartlett's với mức ý nghĩa thống kê (Sig. < 0.001$)$ có ý nghĩa các biến quan sát có mối tương quan với nhau trong tổng thể, trích được hai nhân tố với tổng phương sai trích cho thấy giải thích được $60.387 \%$ sự biến thiên của dữ liệu. Xem xét ma trận nhân tố (Bảng 5) cho thấy biến quan sát PL4 vốn là thành phần của thang đo cảm giác hứng thú lại tải lên cả hai nhân tố và có tỉ lệ hệ số tải $0.427 / 0.503=83 \%>75 \%$ nên phải bỏ để đảm bảo các nhân tố có giá trị phân biệt (Field, 2009; Hair et al., 2010). 


\section{Bảng 5}

Kết quả EFA từng nhân tố của biến trung gian

\begin{tabular}{|l|c|c|c|}
\hline \multicolumn{2}{|c|}{ Thành phần } & \multicolumn{2}{c|}{ Các nhân tố trích } \\
\cline { 3 - 4 } & & $\mathbf{1}$ & $\mathbf{2}$ \\
\hline \multirow{4}{*}{ Cảm giác hứng thú } & PL3 & 0.882 & \\
\cline { 2 - 4 } & PL2 & 0.748 & \\
\cline { 2 - 4 } & PL4 & 0.509 & 0.427 \\
\cline { 2 - 4 } & PL1 & 0.502 & \\
\hline \multirow{3}{*}{ Thôi thúc mua hàng tùy hứng } & UI1 & & 0.815 \\
\cline { 2 - 4 } & UI2 & & 0.713 \\
\cline { 2 - 4 } & UI3 & & 0.692 \\
\hline
\end{tabular}

Nguồn: Kết quả phân tích bằng phần mềm SPSS 25

Kết quả EFA cho biến phụ thuộc cho thấy KMO bằng $0.695(\geq 0.5)$ có ý nghĩa mẫu thích hợp để phân tích nhân tố và kiểm định Bartlett's với mức ý nghĩa thống kê $($ Sig. < 0.001$)$ có ý nghĩa các biến quan sát có mối tương quan với nhau trong tổng thể. Tổng phương sai trích của nhân tố biến phụ thuộc hành vi mua hàng tùy hứng cho thấy giải thích được $64.677 \%$ độ biến thiên của dữ liệu. Ba biến quan sát trong thang đo hành vi mua hàng tùy hứng đều lớn hơn 0.4.

\section{3. Đánh giá giá trị hội tụ và giá trị phân biệt}

Dữ liệu, sau khi đã loại hai biến quan sát MA3 và PL4, được dùng phân tích bằng phần mềm SmartPLS. Trước khi xem xét mô hình cấu trúc tuyến tính, nghiên cứu này xem xét các giá trị hội tụ và phân biệt của thang đo. Giá trị hội tụ sẽ được xác định hệ số tin cậy tổng hợp (CR) và phương sai trích trung bình ( $\mathrm{AVE}$ ), trong đó $\mathrm{CR}$ phải lớn hơn 0.6 và $\mathrm{AVE}$ phải lớn hơn 0.5 (Hair et al., 2010). Kết quả từ SmartPLS cho thấy các thang đo của khái niệm đều đạt yêu cầu về giá trị hội tụ (Bảng 6).

\section{Bảng 6}

Kết quả hệ số tải ngoài, độ tin cậy tổng hợp và phương sai trích trung bình

\begin{tabular}{|c|c|c|c|c|}
\hline \multicolumn{2}{|l|}{ Thành phần } & $\begin{array}{l}\text { Hệ số tải } \\
\text { nhân tố }\end{array}$ & $\begin{array}{l}\text { Độ tin cậy tổng } \\
\text { hợp (CR) }\end{array}$ & $\begin{array}{l}\text { Phương sai trích } \\
\text { trung bình (AVE) }\end{array}$ \\
\hline \multirow{5}{*}{ Nhận biết thương hiệu } & BA1 & 0.778 & \multirow{5}{*}{0.888} & \multirow{5}{*}{0.614} \\
\hline & BA2 & 0.818 & & \\
\hline & BA3 & 0.757 & & \\
\hline & BA4 & 0.751 & & \\
\hline & BA5 & 0.813 & & \\
\hline \multirow{3}{*}{$\begin{array}{l}\text { Trưng bày vật phẩm quảng } \\
\text { cáo tại điểm bán }\end{array}$} & BV1 & 0.825 & \multirow{3}{*}{0.913} & \multirow{3}{*}{0.725} \\
\hline & BV2 & 0.885 & & \\
\hline & BV3 & 0.885 & & \\
\hline
\end{tabular}




\begin{tabular}{|c|c|c|c|c|}
\hline \multicolumn{2}{|l|}{ Thành phần } & $\begin{array}{c}\text { Hệ số tải } \\
\text { nhân tố }\end{array}$ & $\begin{array}{c}\text { Độ tin cậy tổng } \\
\text { hợp (CR) }\end{array}$ & $\begin{array}{l}\text { Phương sai trích } \\
\text { trung bình (AVE) }\end{array}$ \\
\hline & BV4 & 0.808 & & \\
\hline \multirow{3}{*}{ Hành vi mua hàng tùy hứng } & IB1 & 0.904 & \multirow{3}{*}{0.903} & \multirow{3}{*}{0.757} \\
\hline & IB2 & 0.885 & & \\
\hline & IB3 & 0.818 & & \\
\hline \multirow{2}{*}{$\begin{array}{l}\text { Các hoạt động xúc tiến của } \\
\text { điểm bán }\end{array}$} & MA1 & 0.896 & \multirow{2}{*}{0.917} & \multirow{2}{*}{0.847} \\
\hline & MA2 & 0.945 & & \\
\hline \multirow{3}{*}{ Trưng bày sản phẩm } & PD1 & 0.837 & \multirow{3}{*}{0.843} & \multirow{3}{*}{0.642} \\
\hline & PD2 & 0.829 & & \\
\hline & PD3 & 0.733 & & \\
\hline \multirow{3}{*}{ Cảm giác hứng thú } & PL1 & 0.802 & \multirow{3}{*}{0.888} & \multirow{3}{*}{0.726} \\
\hline & PL2 & 0.855 & & \\
\hline & PL3 & 0.897 & & \\
\hline \multirow{3}{*}{ Thôi thúc mua hàng tùy hứng } & UI1 & 0.873 & \multirow{3}{*}{0.904} & \multirow{3}{*}{0.757} \\
\hline & UI2 & 0.888 & & \\
\hline & UI3 & 0.849 & & \\
\hline
\end{tabular}

Nguồn: Kết quả phân tích bằng phần mềm SmartPLS 3.2.9

Giá trị phân biệt của thang đo được đánh giá dựa theo khuyến nghị của Fornell và Larcker (1981) là với từng cặp biến tiềm ẩn thì căn bậc hai cho AVE của mỗi biến tiềm ẩn cao hơn hệ số tương quan giữa hai biến tiềm ẩn của cặp đó. Kết quả phân tích ở Bảng 7 cho thấy căn bậc hai AVE của mỗi nhân tố đều lớn hơn hệ số tương quan giữa nhân tố đó với các nhân tố khác. Như vậy, mẫu nghiên cứu đảm bảo giá trị phân biệt của các thang đo.

\section{Bảng 7}

Kết quả giá trị phân biệt (Fornell-Larcker criterion)

\begin{tabular}{|l|c|c|c|c|c|c|c|}
\hline & BA & BV & IB & MA & PD & PL & UI \\
\hline Nhận biết thương hiệu (BA) & $\mathbf{0 . 7 8 4}$ & & & & & & \\
\hline Trưng bày vật phẩm quảng cáo tại điểm bán (BV) & 0.226 & $\mathbf{0 . 8 5 2}$ & & & & & \\
\hline Hành vi mua hàng tùy hứng (IB) & 0.282 & 0.377 & $\mathbf{0 . 8 7 0}$ & & & & \\
\hline Các hoạt động xúc tiến của điểm bán (MA) & 0.068 & 0.302 & 0.228 & $\mathbf{0 . 9 2 0}$ & & & \\
\hline Trưng bày sản phẩm (PD) & 0.271 & 0.584 & 0.479 & 0.266 & $\mathbf{0 . 8 0 1}$ & & \\
\hline Cảm giác hứng thú (PL) & 0.366 & 0.499 & 0.510 & 0.207 & 0.455 & $\mathbf{0 . 8 5 2}$ & \\
\hline Thôi thúc mua hàng tùy hứng (UI) & 0.277 & 0.396 & 0.658 & 0.225 & 0.429 & 0.587 & $\mathbf{0 . 8 7 0}$ \\
\hline
\end{tabular}

Ghi chú: các số in đậm trên đường chéo là căn bậc hai của AVE

Nguồn: Kết quả phân tích bằng phần mềm SmartPLS 3.2.9 


\section{4. Đánh giá mô hình nghiên cứu}

Kiểm định hiện tượng đa cộng tuyến từ kết quả của SmartPLS đều cho giá trị $\mathrm{VIF}<5$, với giá trị lớn nhất là 2.622, đạt tiêu chí yêu cầu (Henseler et al., 2014) nên mô hình không có vấn đề với đa cộng tuyến. Theo Hu và Bentler (1999) nếu mô hình nhận được giá trị SRMR nhỏ hơn 0.1 thì được xem là phù hợp với dữ liệu thực tế. Với giá trị $\mathrm{SRMR}=0.091<0.1$ thì mô hình nghiên cứu phù hợp với dữ liệu.

Hệ số xác định (giá trị $\mathrm{R}^{2}$ ) được xem xét để đánh giá mô hình cấu trúc. Hair và cộng sự (2010) và Henseler và cộng sự (2014) đã nêu trong nghiên cứu tập trung vào các vấn đề tiếp thị, các giá trị của $\mathrm{R}^{2}$ ở $0.75 ; 0.50$ hoặc 0.25 cho các biến tiềm ẩn nội sinh được coi là tương ứng đáng kể, trung bình hoặc yếu. Tuy nhiên, giá trị $\mathrm{R}^{2}=0.20$ được xem là cao trong nghiên cứu hành vi người tiêu dùng (Hair et al., 2010). Kết quả cho thấy các hệ số $\mathrm{R}^{2}$ và $\mathrm{R}^{2}$ hiệu chỉnh của biến phụ thuộc cuối cùng là hành vi mua hàng tùy hứng $(\mathrm{IB})$ tương ứng là 0.433 và 0.431 , cho thấy các mối quan hệ trong mô hình nghiên cứu giải thích được khoảng $43 \%$ phương sai của hành vi mua hàng tùy hứng.

Nghiên cứu cũng đánh giá tầm quan trọng của các giá trị $\mathrm{R}^{2}$ bằng kiểm tra giá trị $\mathrm{Q}^{2}$. Kết quả cho thấy hành vi mua hàng tùy hứng (IB) có giá trị $\mathrm{Q}^{2}$ cao nhất $(0.322)$, tiếp theo là Thôi thúc mua hàng tùy hứng UI $(0.257)$ và cảm giác hứng thú $(\mathrm{PL})(0.241)$, chứng tỏ có sự liên quan dự báo của mô hình đến các biến tiềm ẩn nội sinh.

\subsection{Kiểm định giả thuyết nghiên cứu}

Kết quả nghiên cứu đã chỉ ra ba yếu tố marketing tại điểm bán tác động đến hành vi mua hàng tùy hứng thực phẩm đóng gói là trưng bày vật phẩm quảng cáo tại điểm bán, nhận biết thương hiệu, trưng bày sản phẩm (Bảng 8 ). Kết quả này, trừ $\mathrm{H} 2$ bị bác bỏ, tương đồng với các nghiên cứu của Zhou và Wong (2004), Bellini và cộng sự (2017), Iyer và cộng sự (2020) khi nói về vai trò của các yếu tố marketing tại điểm bán tác động đến hành vi mua hàng tùy hứng. Kết quả H2 không được chấp nhận như kỳ vọng có thể do trong bối cảnh nghiên cứu một món hàng thực phẩm đóng gói có giá trị thấp và số tiền mua tùy hứng thấp nên người mua không quan trọng đến khuyến mãi giảm giá hay sự tích cực giới thiệu của nhân viên. Ba yếu tố tác động đến hành vi mua hàng tùy hứng thông qua phản ứng tâm lý trong tâm trí của khách hàng lần lượt là cảm giác hứng thú và thôi thúc mua hàng tùy hứng. Kết quả này tương đồng với các nghiên cứu của Fiore và cộng sự (2000), Beatty và Ferrell (1998), Choi (2016) và Bandyopadhyay (2016).

\section{Bảng 8}

Kết quả kiểm định các hệ số mô hình cấu trúc

\begin{tabular}{|c|c|c|c|c|c|c|c|}
\hline $\begin{array}{c}\text { Giả } \\
\text { thuyết }\end{array}$ & $\begin{array}{c}\text { Mối } \\
\text { quan hệ }\end{array}$ & $\begin{array}{c}\text { Hệ số } \\
\text { hồi quy }\end{array}$ & $\begin{array}{c}\text { Hệ số hồi quy } \\
\text { Bootstrapping }\end{array}$ & $\begin{array}{c}\text { Độ lệ̉ch } \\
\text { chuẩn }\end{array}$ & Giá trị t & $\begin{array}{c}\text { Giá trị } \\
\text { p }\end{array}$ & Kết quả (*) \\
\hline H1 & PD $\rightarrow$ PL & 0.192 & 0.194 & 0.065 & 2.954 & 0.003 & Chấp nhận \\
\hline H2 & MA $\rightarrow$ PL & 0.042 & 0.043 & 0.052 & 0.811 & 0.418 & Bác bỏ \\
\hline H3 & BV $\rightarrow$ PL & 0.320 & 0.319 & 0.066 & 4.863 & 0.000 & Chấp nhận \\
\hline H4 & BA $\rightarrow$ PL & 0.239 & 0.244 & 0.058 & 4.100 & 0.000 & Chấp nhận \\
\hline H5 & PL $\rightarrow$ UI & 0.587 & 0.588 & 0.044 & 13.430 & 0.000 & Chấp nhận \\
\hline H6 & UI $\rightarrow$ IB & 0.658 & 0.660 & 0.046 & 14.232 & 0.000 & Chấp nhận \\
\hline
\end{tabular}

Ghi chú: PD: Trưng bày sản phẩm; PL: Cảm giác hứng thú; MA: Các hoạt động xúc tiến của điểm bán; BV: Trưng bày vật phẩm quảng cáo tại điểm bán; $\mathrm{BA}$ : Nhận biết thương hiệu; UI: Thôi thúc mua hàng tùy hứng; IB: hành vi mua hàng tùy hứng; $(*)$ Alpha $=0.05$

Nguồn: Kết quả phân tích bằng phần mềm SmartPLS 3.2.9 
Với việc các giả thuyết H1, H3, H4 và H5 được chấp nhận, thì theo như Kenny (2008), có thể lập luận rằng cảm giác hứng thú đóng vai trò trung gian từ các yếu tố marketing là trưng bày vật phẩm quảng cáo tại điểm bán, nhận biết thương hiệu, và trưng bày sản phẩm đến thôi thúc và cuối cùng là hành vi mua hàng tùy hứng. Theo Kenny (2008) thì không cần thiết đánh giá trung gian từng phần hay toàn phần. Bên cạnh đó, Preacher và Hayes (2008) cũng chỉ ra rằng có thể dùng bootstrapping để đánh giá ý nghĩa thống kê của mối quan hệ gián tiếp, và trong nghiên cứu này PLS-SEM đã ước lượng các giá trị $p$ dựa trên bootstrapping.

\section{Kết luận, đóng góp và hàm ý của nghiên cứu, và hướng nghiên cứu tương lai}

Kết quả nghiên cứu đóng góp phát triển lý thuyết về hành vi người tiêu dùng, và cụ thể là hành vi mua hàng tùy hứng. Nếu như các nghiên cứu trước đây cho thấy loại sản phẩm có ảnh hưởng đến hành vi mua hàng tùy hứng thì nghiên cứu này làm rõ thêm là với mỗi loại sản phẩm khác nhau thì các yếu tố marketing tại điểm bán có ảnh hưởng khác nhau hay thậm chí không ảnh hưởng đến hành vi mua hàng tùy hứng. Cụ thể trong bối cảnh mua tùy hứng một món hàng thực phẩm đóng gói mà hóa đơn mua hàng tùy hứng không cao thì các yếu tố liên quan đến không gian điểm bán lẻ như trưng bày, đặt để vật phẩm quảng cáo (như áp phích quảng cáo, standee quảng cáo ...) sản phẩm hay logo để người mua nhận diện hay gợi nhớ có thể làm tăng khả năng gợi cảm xúc, thôi thúc mua tùy hứng và cuối cùng là hành vi mua hàng tùy hứng. Trong khi, phần lớn các nghiên cứu trước đây tập trung các ngành thời trang, mỹ phẩm, ... là loại hàng/tình huống mua hàng mà khách hàng có thể bận tâm cao (high-involvement) khi mua hàng (Rossiter et al., 1991) hay còn được xếp là loại hàng mua sắm (shopping products) (Kotler \& Keller, 2016), thì nghiên cứu tập trung vào loại sản phẩm tiện lợi (convenience products), tình huống mua hàng đòi hỏi mức độ bận tâm thấp (Kotler \& Keller, 2016; Rossiter et al., 1991) có hiếm hoi nghiên cứu trước được công bố. Có thể, đây là một lý do có thể khi mà kết quả của nghiên cứu này cho thấy các hoạt động xúc tiến tại điểm bán với các hoạt động như là khuyến mãi, tặng kèm không có tác động đến hành vi mua hàng tùy hứng do giá trị thấp của hóa đơn mua hàng tùy hứng hàng tiện lợi. Bên cạnh đó, nghiên cứu này còn cung cấp thêm từ bối cảnh thị trường Việt Nam là một thị trường mới nổi, đóng góp minh chứng cho mảng nghiên cứu quan trọng về thị trường mới nổi trong marketing.

Về mặt thực tiễn, các doanh nghiệp có thể dựa trên kết quả nghiên cứu để xây dựng chiến lược marketing tại điểm bán cho mặt hàng thực phẩm đóng gói và có thể mở rộng ra các mặt hàng tiện lợi/hàng tiêu dùng nhanh để thúc đẩy hành vi mua hàng tùy hứng tại điểm bán. Trong bối cảnh mặt hàng thực phẩm đóng gói, các doanh nghiệp trong ngành hàng thực phẩm đóng gói cần xem xét đầu tư cho các hoạt động marketing tại điểm bán như trưng bày các vật phẩm quảng cáo, hay đặt/để logo để người tiêu dùng dễ thấy hay trưng bày sản phẩm tại điểm bán. Kết quả của nghiên cứu này trong đó tác động mạnh nhất là từ yếu tố trưng bày vật phẩm quảng cáo tại điểm bán, kế đến là sự nhận biết cho thấy tầm quan trọng của truyền thông marketing tại điểm bán hướng đến người mua, chứ không chỉ khách hàng hay người tiêu dùng nói chung. Như vậy, bên cạnh các hoạt động truyền thông để xây dựng nhận biết và hình ảnh thương hiệu qua phương tiện đại chúng thì doanh nghiệp cũng phải chú trọng duy trì sự hiện diện của nhận diện thương hiệu và các công cụ truyền thông tại điểm bán. Thứ nhất, để thực hiện hiệu quả hoạt động trưng bày vật phẩm quảng cáo tại điểm bán, các doanh nghiệp cần chú ý bốn yêu cầu sau đối với một vật phẩm quảng cáo tại điểm bán: Chứa đựng thông tin, thu hút khách hàng mục tiêu, có thể mang lại cảm giác thú vị và hiện đại. Thứ hai, nâng cao mức độ nhận biết thương hiệu không chỉ là một hoạt động qua truyền thông đại chúng mà doanh nghiệp cũng cần phải làm thế nào ở tại điểm bán có thể giúp khách hàng nhận ra thương hiệu của mình giữa rất nhiều thương hiệu khác cạnh tranh. Thứ ba, đối với hoạt động trưng bày sản phẩm, trưng bày hiệu quả cần đảm bảo các 
yếu tố: hấp dẫn, bố trí ở những vị trí dễ thấy và độc chiếm. Cuối cùng và quan trọng nhất là các hoạt động marketing tại điểm bán trên phải đáp ứng yêu cầu là gợi cảm giác hứng thú và thúc đẩy người mua chọn lựa sản phẩm/thương hiệu khi mua hàng tùy hứng.

Như mọi nghiên cứu khác, nghiên cứu này không tránh khỏi hạn chế. Thứ nhất, kết quả nghiên cứu từ khảo sát tại một thời điểm, nên trong tương lai có thể kết hợp các phương pháp/thiết kế khác để tăng khả năng khái quát hóa kết quả. Thứ hai, so với kế hoạch ban đầu tập trung khảo sát trực tiếp tại các điểm bán lẻ ở TPHCM để đảm bảo đáp viên trả lời ngay khi vừa mua hàng tùy hứng, nhưng do đại dịch coronavirus nên việc lấy mẫu phải chuyển một phần sang trực tuyến, do đó dù đã có câu hỏi lọc nhưng có thể không tránh khỏi những hạn chế như bất kỳ phương pháp nghiên cứu qua Internet nào như đáp viên có thể quan tâm nhiều hơn về hiện tượng nghiên cứu hay sai biệt phương pháp chung. Trong tương lai, nghiên cứu sẽ xem xét thêm các yếu tố khác ngoài môi trường nhưng cũng liên quan đến thời điểm/không gian điểm bán lẻ khi hành vi mua tùy hứng được thực hiện như sự hiện diện của các khách hàng khác. Mở rộng phạm vi nghiên cứu ra các tỉnh/thành khác của Việt Nam sẽ tạo ra tính khái quát cao hơn cho nghiên cứu.

\section{Tài liệu tham khảo}

Aaker, D. A. (1996). Measuring brand equity across products and markets. California Management Review, 38(3), 102-120.

Abratt, R., \& Goodey, S. D. (1990). Unplanned buying and in-store stimuli in supermarkets. Managerial and Decision Economics, 11(2), 111-121.

Amos, C., Holmes, G. R., \& Keneson, W. C. (2014). A meta-analysis of consumer impulse buying. Journal of Retailing and Consumer Services, 21(2), 86-97.

Atulkar, S., \& Kesari, B. (2018). Role of consumer traits and situational factors on impulse buying. Does gender matter? International Journal of Retail and Distribution Management, 46(4), 386-405.

Badgaiyan, A. J., \& Verma, A. (2014). Intrinsic factors affecting impulsive buying behaviourEvidence from India. Journal of Retailing and Consumer Services, 21(4), 537-549.

Bandyopadhyay, N. (2016). The role of self-esteem, negative affect and normative influence in impulse buying: A study from India. Marketing Intelligence và Planning, 34(4), 523-539.

Bearden, W. O., \& Netemeyer, R. G. (1999). Handbook of marketing scales: Multi-item measures for marketing and consumer behavior research. London, UK: Sage Publications.

Beatty, S. E., \& Ferrell, M. E. (1998). Impulse buying: Modeling its precursors. Journal of Retailing, 74(2), 169-191.

Bell, D., Corsten, D., \& Knox, D. (2011). From point of purchase to path to purchase. How preshopping factors drive unplanned buying. Journal of Marketing, 75(1), 31-45.

Bellini, S., Cardinali, M. G., \& Grandi, B. (2017) A structural equation model of impulse buying behaviour in grocery retailing. Journal of Retailing and Consumer Services, 36, 164-171.

Bigné, J. E., Andreu, L., \& Gnoth, J. (2005). The theme park experience: An analysis of pleasure, arousal and satisfaction. Tourism Management, 26(6), 833-844.

Chang, H. J., Eckman, M., \& Yan, R. N. (2011). Application of the stimulus-organism-response model to the retail environment: The role of hedonic motivation in impulse buying 
behavior. The International Review of Retail, Distribution and Consumer Research, 21(3), 233-249.

Chen, H. T., \& Lin, Y. T. (2018). A study of the relationships among sensory experience, emotion, and buying behavior in coffeehouse chains. Service Business, 12(3), 551-573.

Choi, J. (2016). Consumer impulse buying of food at festivals and events understanding the role of sensory cues (Doctoral dissertation). Kansas State University, Manhattan, Kansas, USA.

Coley, A., \& Burgess, B. (2003). Gender differences in cognitive and affective impulse buying. Journal of Fashion Marketing and Management, 7(3), 282-295.

Field, A. (2009). Discovering statistics using SPSS (and sex and drugs and rock ' $n$ ' roll) (3rd ed.). London, UK: Sage.

Fiore, A. M., Yah, X., \& Yoh, E. (2000). Effects of a product display and environmental fragrance on approach responses and pleasurable experiences. Journal of Psychology and Marketing, 17(1), 27-54.

Fornell, C., \& Larcker, D. F. (1981). Evaluating structural equation models with unobservable variables and measurement error. Journal of Marketing Research, 18(1), 39-50.

Hair, J. F., Anderson, R. E., Babin, B. J., \& Black, W. C. (2010). Multivariate data analysis: A global perspective. Upper Saddle River, NJ: Pearson.

Henseler, J., Dijkstra, T. K., Sarstedt, M., Ringle, C. M., Diamantopoulos, A., Straub, D. W., ... Calantone, R. J. (2014). Common beliefs and reality about PLS: Comments on Rönkkö and Evermann (2013). Organizational Research Methods, 17(2), 182-209.

Hu, L. T., \& Bentler, P. M. (1999). Cut off criteria for fit indexes in covariance structure analysis: Conventional criteria versus new alternatives. Structural Equation Modeling: A Multidisciplinary Journal, 6(1), 1-55.

Inman, J. J., Winer, R. S., \& Ferraro, R. (2009). The interplay among category characteristics, customer characteristics, and customer activities on in-store decision making. Journal of Marketing, 73(5), 19-29.

Iyer, G. R., Blut, M., Xiao, S. H., \& Grewal, D. (2020). Impulse buying: A meta-analytic review. Journal of the Academy of Marketing Science, 48(3), 384-404.

Jones, M. A., Reynolds, K. E., Weun, S., \& Beatty, S. E. (2003). The product-specific nature of impulse buying tendency. Journal of Business Research, 56(7), 505-511.

Kacen, J. J., \& Lee, J. A. (2002). The influence of culture on consumer impulsive buying behavior. Journal of Consumer Psychology, 12(2), 163-176.

Keller, K. L., Parameswaran, M. G., \& Jacob, I. (2011). Strategic brand management: Building, measuring, and managing brand equity. Delhi, India: Pearson Education.

Kenny, D. A. (2008). Reflections on mediation. Organizational Research Methods, 11(2), 353-358.

Kim, M. J., Lee, C. K., \& Jung, T. (2020). Exploring consumer behavior in virtual reality tourism using an extended stimulus-organism-response model. Journal of Travel Research, 59(1), 69-89.

Kotler, P., \& Keller, K. L. (2016). Marketing management (15th ed.). London, UK: Pearson.

Lee, J. (2008). Relative and interaction effects of situational and personal factors on impulse buying (Doctoral dissertation). University of Minnesota, Minnesota, USA. 
Leith, K. P., \& Baumeister, R. F. (1996). Why do bad moods increase self-defeating behavior? Emotion, risk tasking, and self-regulation. Journal of Personality and Social Psychology, 71(6), 1250-1267.

Mehrabian, A., \& Russell, J. A. (1974). An approach to environmental psychology. Cambridge, MA: MIT Press.

Mishra, N. (2012). A study on impulse buying behavior of working men and women in Delhi/NCR. Anvesha, 5(3), Article 23.

Mohan, G., Sivakumaran, B., \& Sharma, P. (2013). Impact of store environment on impulse buying behavior. European Journal of Marketing, 47(10), 1711-1732.

Nguyen, H. M. (2014). Nghiên cứu quyết định mua và sụ lựa chọn của khách hàng [Researching customers' purchasing decisions and choices]. Ho Chi Minh City, Vietnam: Nhà Xuất bản Kinh tế Thành phố Hồ Chí Minh.

Nguyen, H. M., \& Vu, T. H. (2020). Phân tích dĩu liệu áp dụng mô hình PLS - SEM [Data analysis using PLS - SEM model]. Ho Chi Minh City, Vietnam: Nhà Xuất bản Kinh tế Thành phố Hồ Chí Minh.

Palazon, M., \& Ballester, E. D. (2011). The expected benefit as determinant of deal-prone consumers' response to sales promotions. Journal of Retailing and Consumer Services, $18(6), 542-547$.

Park, E. J., Kim, E. Y., \& Forney, J. C. (2006). A structural model of fashion oriented impulse buying behavior. Journal of Fashion Marketing and Management, 10(4), 433-446.

Parsad, C., Prashar, S., \& Tata, V. S. (2017). Understanding nature of store ambiance and individual impulse buying tendency on impulsive purchasing behaviour: An emerging market perspective. Decision, 44(4), 297-311.

Peck, J., \& Childers, T. L. (2006). If I touch it I have to have it: Individual and environmental influences on impulse purchasing. Journal of Business Research, 59(6), 765-769.

Poels, K., \& Dewitte, S. (2008). Getting a line on print ads: Pleasure and arousal reactions reveal an implicit advertising mechanism. Journal of Advertising, 37(4), 63-74.

Prashar, S., Parsad, C., Tata, S. V., \& Sahay, V. (2015). Impulsive buying structure in retailing: An interpretive Structural modeling approach. Journal of Marketing Analytics, $3(4), 215-233$.

Preacher, K. J., \& Hayes, A. F. (2008). Asymptotic and resampling strategies for assessing and comparing indirect effects in multiple mediator models. Behavior Research Methods, 40(3), 879-891.

Robert, D., \& John, R. (1982). Store atmosphere an environmental psychology approach. Journal of Retailing, 58(1), 34-57.

Rook, D. W. (1987). The buying impulse. Journal of Consumer Research, 14(2), 189-197.

Rook, D. W., \& Fisher, R. J. (1995). Normative influences on impulsive buying behavior. Journal of Consumer Research, 22(3), 305-313.

Rook, D. W., \& Gardner, M. P. (1993). In the mood: Impulse buying's affective antecedents. Research in Consumer Behavior, 6(7), 1-28. 
Rossiter, J. R., Percy, L., \& Donovan, R. J. (1991). A better advertising planning grid. Journal of Advertising Research. 31(5), 11-21.

Ryu, K., \& Jang, S. S. (2007). The effect of environmental perceptions on behavioral intentions through emotions: The case of upscale restaurants. Journal of Hospitality \& Tourism Research, 31(1), 56-72.

Santini, F. D. O., Ladeira, W. J., Vieira, V. A., Araujo, C. F., \& Sampaio, C. H. (2019). Antecedents and consequences of impulse buying a meta-analytic study. RAUSP Management Journal, 54(2), 178-204.

Sheth, J. N. (2011). Impact of emerging markets on marketing: Rethinking existing perspectives and practices. Journal of Marketing, 75(4), 166-182.

Sorensen, H., Bogomolova, S., Anderson, K., Trinh, G., Sharp, A., Kennedy, R., ... Wright, M. (2017). Fundamental patterns of in-store shopper behavior. Journal of Retailing and Consumer Services, 37, 182-194.

Weun, S., Jones, M. A., \& Beatty, S. E. (1998). Development and validation of the impulse buying tendency scale. Psychological Reports, 82(3), 1123-1133.

Wu, L., Chen, K. W., \& Chiu, M. L. (2016). Defining key drivers of online impulse purchasing: A perspective of both impulse shoppers and system users. International Journal of Information Management, 36(3), 284-296.

Wu, P. T., \& Lee, C. J. (2016). Impulse buying behaviour in cosmetics marketing activities. Total Quality Management and Business Excellence, 27(9/10), 1091-1111.

Zhou, L., \& Wong, A. (2004). Consumer impulse buying and in-store stimuli in Chinese supermarkets. Journal of International Consumer Marketing, 16(2), 37-53. 